\title{
Maternal Risk Factors of Premature Rupture of Membrane (PROM) after 37 Weeks of Pregnancy
}

\section{ART ICLE INFO}

\section{Article Type}

Original research

\section{Authors}

Alijani L. ${ }^{1} M D$,

Mosadegh H. ${ }^{1} M D$,

Saremi A.T.* $M D$

Mashhadikhan M. ${ }^{2} \mathrm{PhD}$

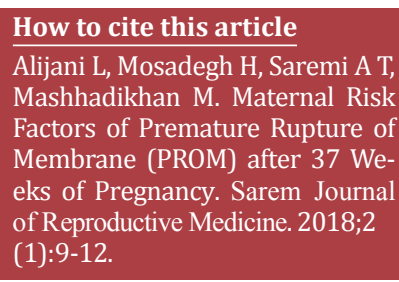

*"Sarem Fertility \& Infertility Research Center (SAFIR)" and "Sarem Cell Research Center (SCRC)", Sarem Women's Hospital, Tehran, Iran ${ }^{1}$ Sarem Fertility \& Infertility Research Center (SAFIR), Sarem Women's Hospital, Tehran, Iran

${ }^{2}$ Sarem Cell Research Center (SCRC), Sarem Women's Hospital, Tehran, Iran

\section{Correspondence}

Address: Sarem Women's Hospital, Basij Square, Phase 3, Ekbatan Town, Tehran, Iran. Postal Code: 1396956111

Phone: +98 (21) 44670888

Fax: +98 (21) 44670432

saremiat@yahoo.com

\section{Article History}

Received: September 23, 2016

Accepted:January 12, 2017

ePublished: February 15, 2018

\section{A B S T R A C T}

Aims Different risk factors are known to be contributed to premature rupture of membrane (PROM). However, its certain cause has always been discussed. Therefore, the present study was aimed to investigate the maternal risk factors of PROM after 37 weeks of pregnancy. Materials \& Methods This case-control study was conducted on 242 pregnant women referred to Sarem women's hospital (Tehran, Iran) in 2011-2013. The women in case group (n=121) had PROM at least one hour before delivery. The women in control group $(n=121)$ were matched in terms of maternal age with case group. The effects of factors including vaginal culture, abortion history, gravids, history of cervical surgery and cervical length were investigated on PROM. Data were analyzed using Chi-square, Fischer exact and Mann-Whitney tests by SPSS software. Findings There was no significant difference in age distribution between case and control groups. There were no significance associations between gravids, vaginal culture and history of cerclage with PROM ( $p>0.05)$. However, significant associations were found between the numbers of previous abortions and cervix length less than $35 \mathrm{~mm}$ with PROM $(\mathrm{p}<0.05)$.

Conclusion The factors including cervical length less than $35 \mathrm{~mm}$ and the number of previous abortions (both of which are related in some way) are the maternal risk factors affecting PROM after 37 weeks of pregnancy.

Keywords Vaginal Discharge; Gestational Age; Abortion; Cervix; Cerclage

\section{I T A T I O N L I N K S}

[1] Evaluation of pregnant women admitted with prelabour rupture of membranes (PROM) [2] Neonatal complications of premature rupture of membrane [3] Cause analysis and clinical management experience of the premature rupture of membrane [4] Study of patient with premature rupture of membranes [5] Creasy and resnik's maternal-fetal medicine: Principles and practice [6] Premature rupture of membranes characteristics, determinants, and outcomes of in Benghazi, Libya [7] Pre and post conception risk factors in PROM [8] Neonatal complications related with prolonged rupture of membranes [9] Investigation of risk factors according to gestational age in cases with premature rupture of membrane [10] Diagnosis of premature rupture of membranes: Inspiration from the past and insights for the future [11] Guidelines for the management of spontaneous preterm labor: Identification of spontaneous preterm labor, diagnosis of preterm premature rupture of membranes, and preventive tools for preterm birth [12] Clinical and inflammatory markers in amniotic fluid as predictors of adverse outcomes in preterm premature rupture of membranes [13] Preterm premature rupture of membranes: Risks of recurrent complications in the next pregnancy among a population-based sample of gravid women [14] Analysis of some maternal risk factors in preterm delivery [15] The prevention, diagnosis and treatment of premature labor [16] Emergency cerclage: A review [17] Cerclage for short cervix on ultrasonography in women with singleton gestations and previous preterm birth: A meta-analysis [18] Risk factors for prematurity and premature rupture of membranes: A prospective study of the vaginal flora in pregnancy [19] Antimicrobial therapy in expectant management of preterm premature rupture of the membranes [20] Antibiotics for preterm rupture of membranes [21] Neonatal morbidity at 34-37 weeks: The role of ruptured membranes 


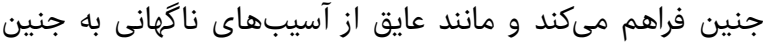

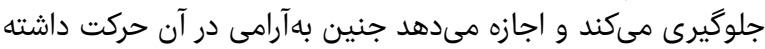

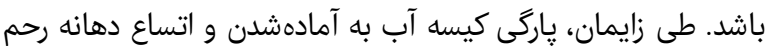

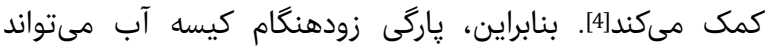

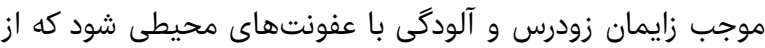

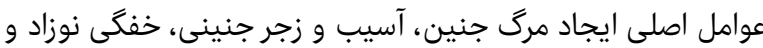

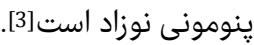

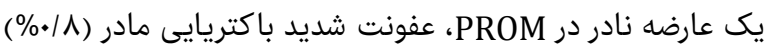

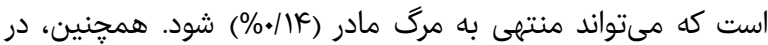

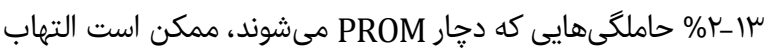

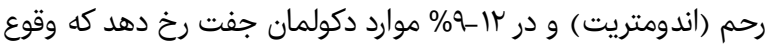

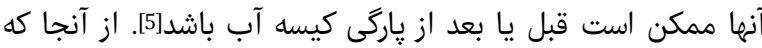

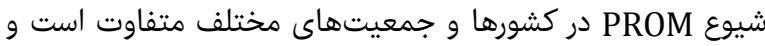

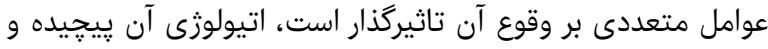

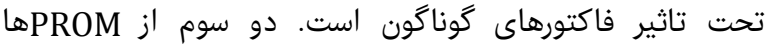

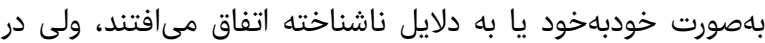

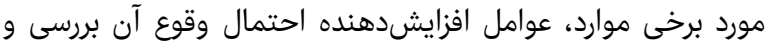

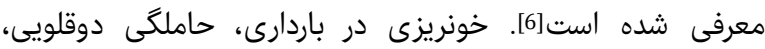

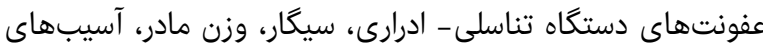

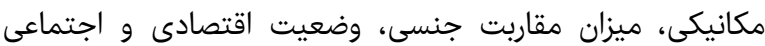

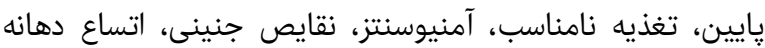

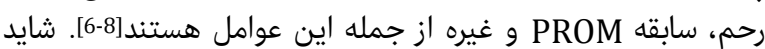

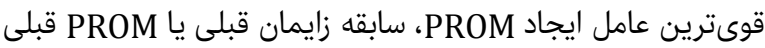

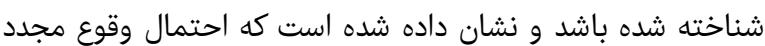

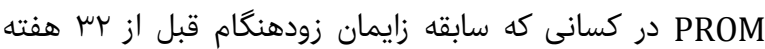
(early perterm)

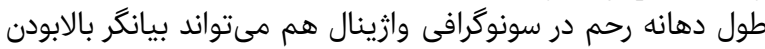

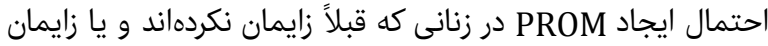

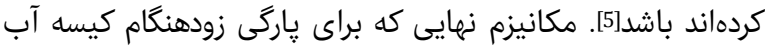

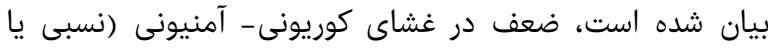

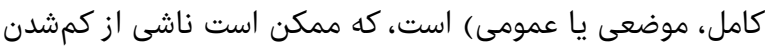

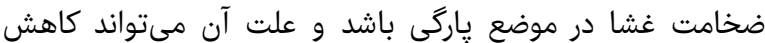

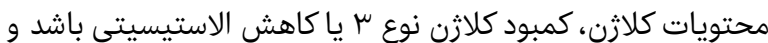

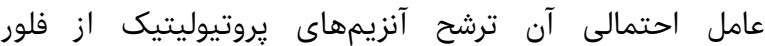

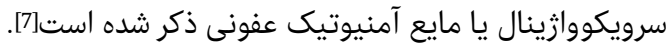

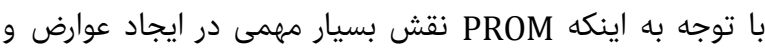

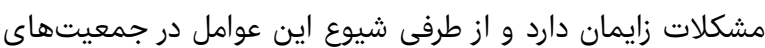

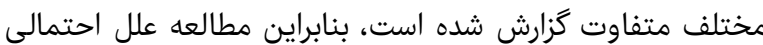

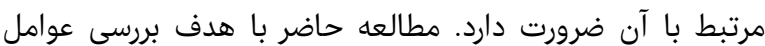

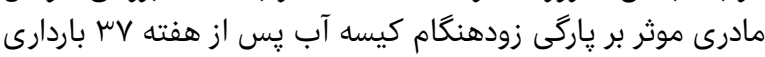

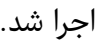

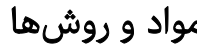

اين مطالعه مورد- شاهدى روى موى زنان باردار مراجعهكننده به

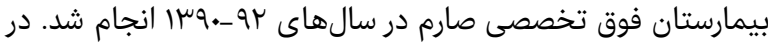

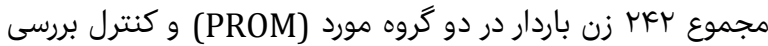

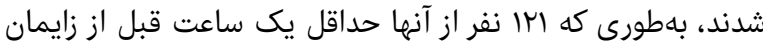

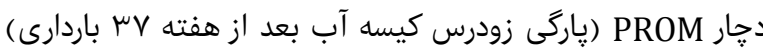

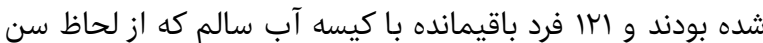

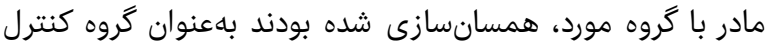

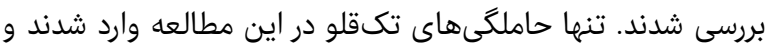

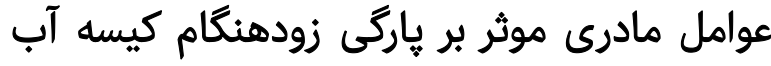

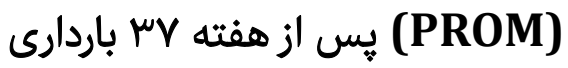

ليلا عليجانى

مركز تحقيقات بارورى و نابارورى صارم، بيمارستان فوق تخصصى صارم، تهران،

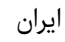

حميرا مصدق ايران مركز تحقيقات بارورى و نابارورى صارم، بيمارستان فوق تخصصى صارم، تهران،

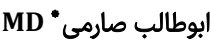

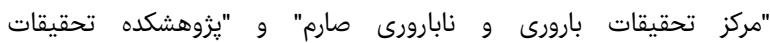
سلولى-مولكولى و سلولهاى بنديادى صاروى صارم"، بيمارستان فوق تخصصى صارم،

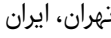

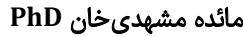

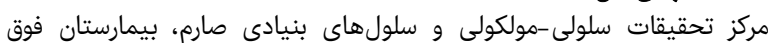
تخصصى صارم، تهران، ايران

جكيده

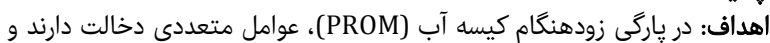

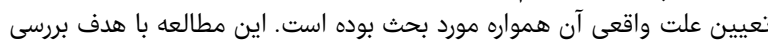

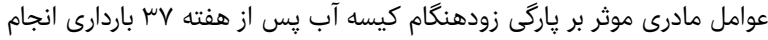

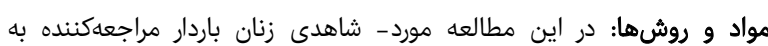

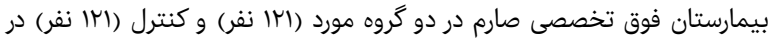

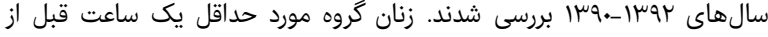

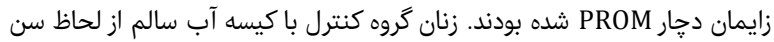

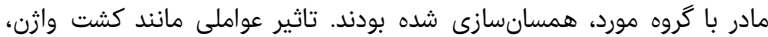

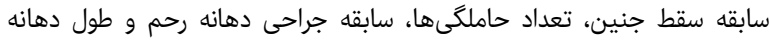

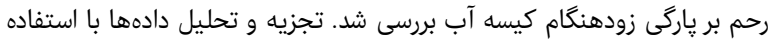

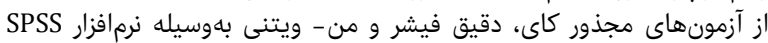

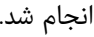

يافتهها: توزيع فراوانى سنى بين دو كروه مورد و كنترل تفاوت معات معنىدارى دارى

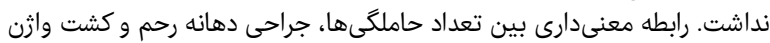

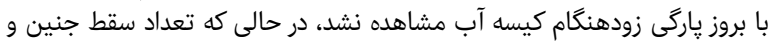

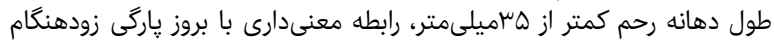

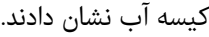

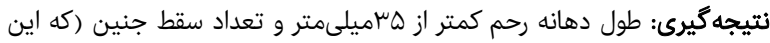

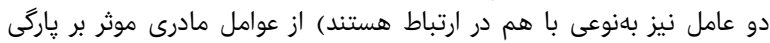

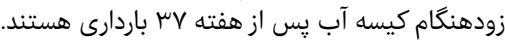
كليدوازهها: كشت واثن، سن باردارى، سقط جنين، دهانه رحم، سركلاز

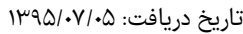

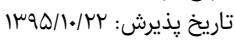
"نويسنده مسئول: Saremiat@yahoo.com

مقدمه

به ياركى زودهنكام كيسه آب جنين قبل از شروع دردهاى زايمان و قبل از

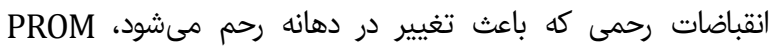

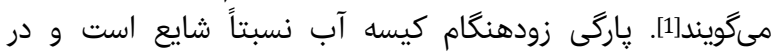

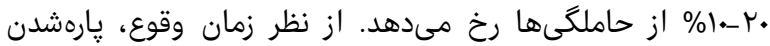

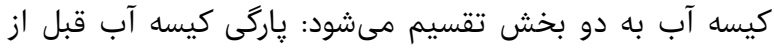

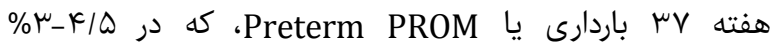

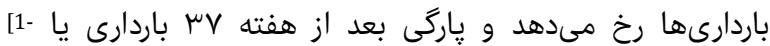
.31PROM باردارئ

كيسه آب نقشهاى مختلفى دارد كه يِيشگيرى از انتقال عمودى

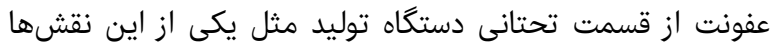
است. همجنين كيسه آب، محيطى با دمايى يكنواخت در إنى اطراف نقاف 


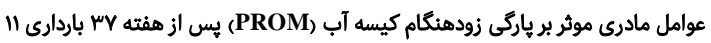

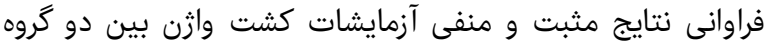

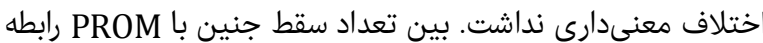

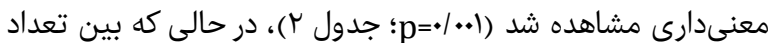

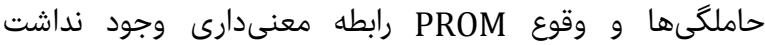

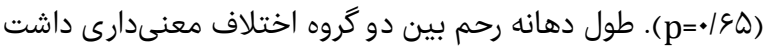

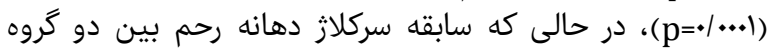

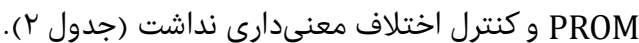

بحث

در مطالعات متعددى نقش عوامل مختلف مادرى و حتى جنينى

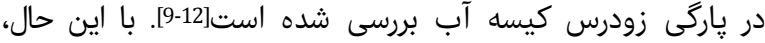

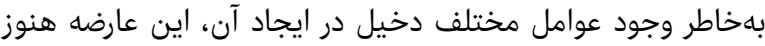

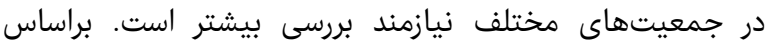

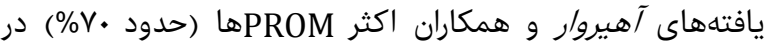

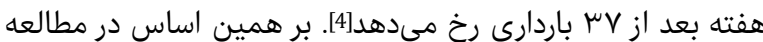

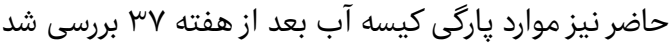

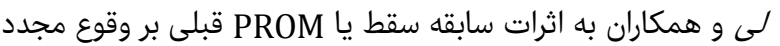

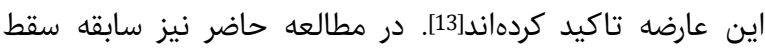

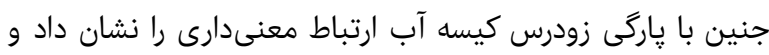

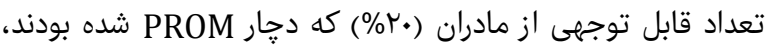

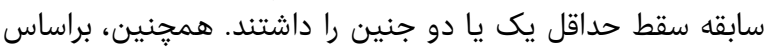

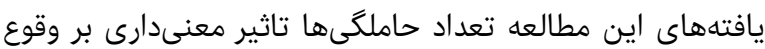

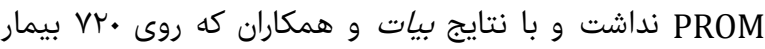

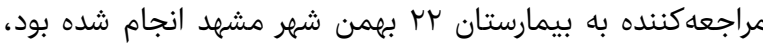
همخوانى داشت[14].

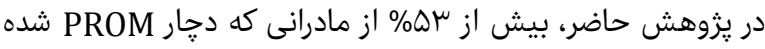

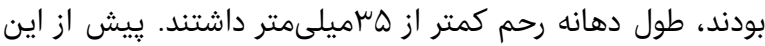

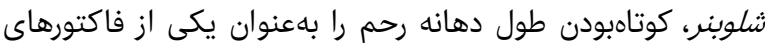

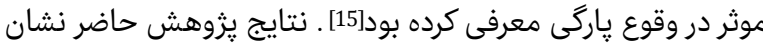

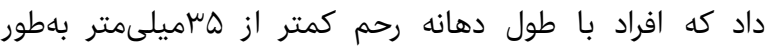

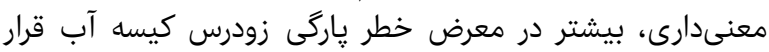

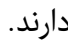
در مطالعه مرورى آرتز و همكاران[16] و همجنين در مطار مطالعه برقلا و

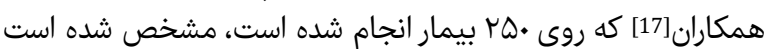

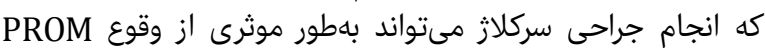

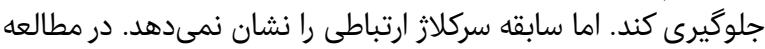
حاضر نيز سابقه جراحى سركلاز در افرادى كه دجار

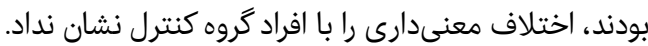

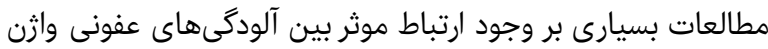

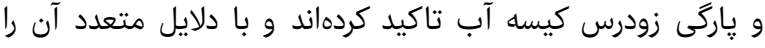

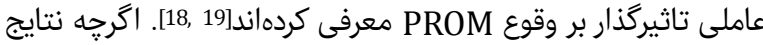

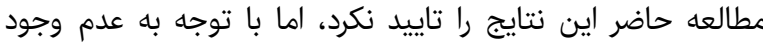

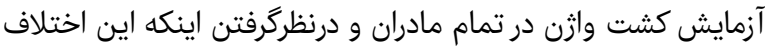

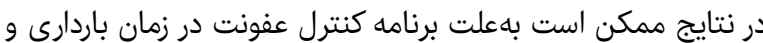

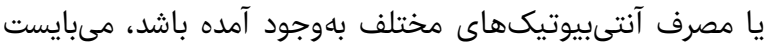

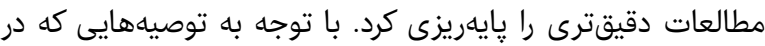

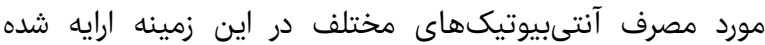

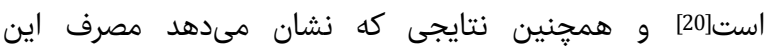

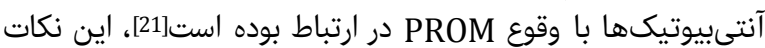

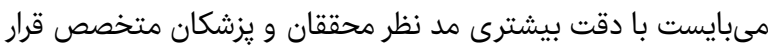

نوزادان با تشخيص ناهنجارى از مطالعه حذف شدند.

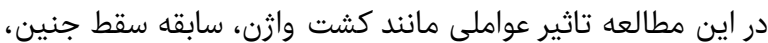

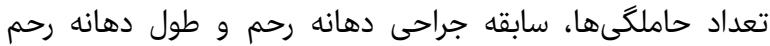

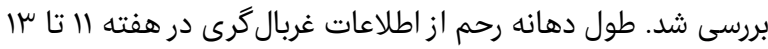

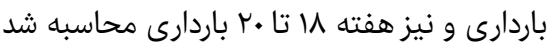

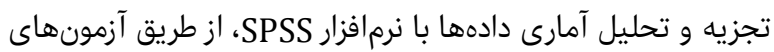

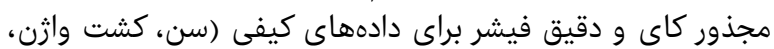

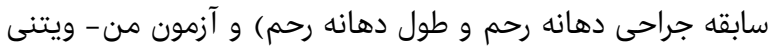

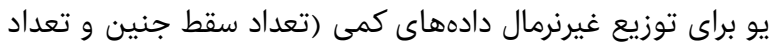
حاملگىها) صورت كرفت.

يافتهها

بيشترين فراوانى مادران در هر دو گروه مورد (PROM) و كنترل در درا

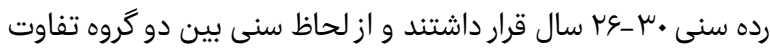

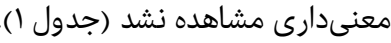

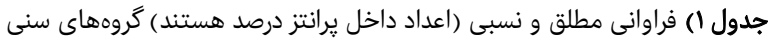

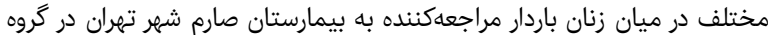

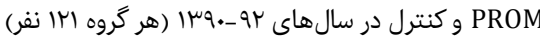

\begin{tabular}{|c|c|c|}
\hline 3روه كنترل & Fروه PROM & رده سنى مادران" \\
\hline$(1 \Lambda / K) \mathrm{Kr}$ & $(11 / 9) \|^{k}$ & •r تا كזسال \\
\hline$(K Y / I) Q 1$ & $(\Delta r / I) \& \mu$ & عץ تا •باسال \\
\hline$(\mu \mu / l) k$. & $(r \wedge / q) r \Delta$ & اس تا هسال \\
\hline$(\varepsilon / \varepsilon) \wedge$ & $(V / F)^{9}$ & بيش از عسال \\
\hline
\end{tabular}

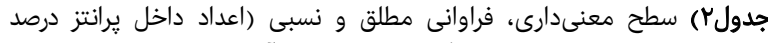

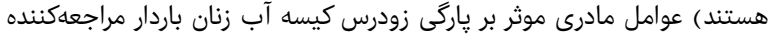

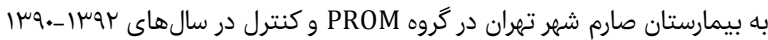

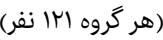

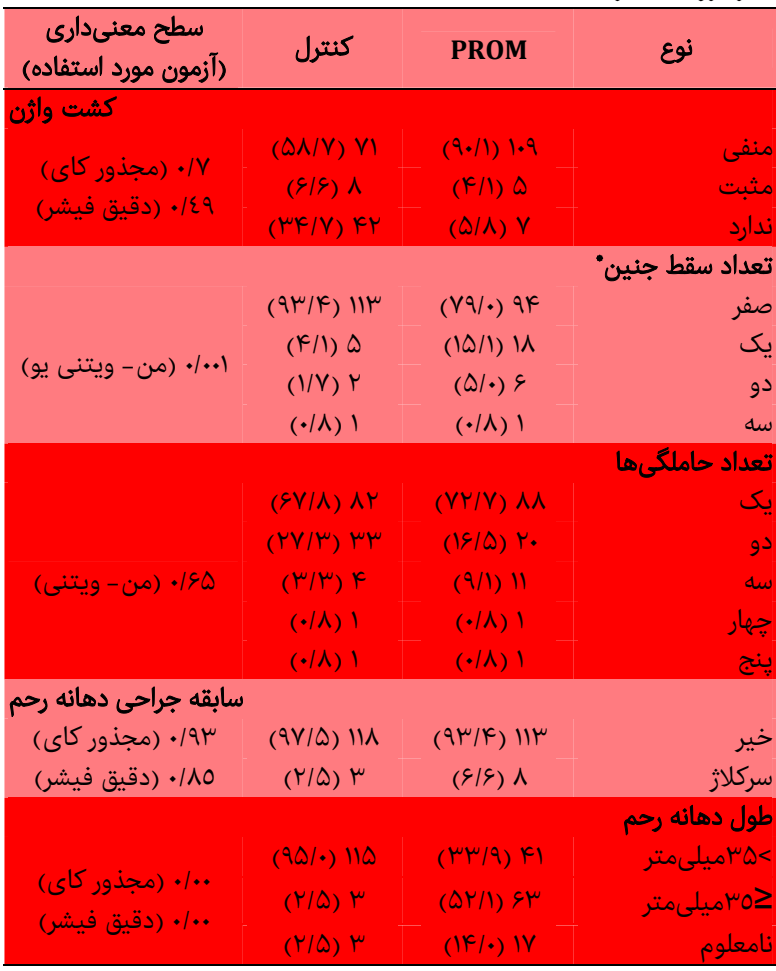

دمجموع فراوانى در گروه PROM براى متغير تعداد سقط جنين (19 نفر) مربوط به

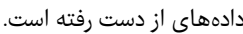


2015;3(10):2594-8.

8- Boskabadi H, Maamouri G, Mafinejad S. Neonatal complications related with prolonged rupture of membranes. Maced J Med Sci. 2011;4(1):93-8.

9- Yildiz $\mathrm{H}$, Abali S, Keskin S. Investigation of risk factors according to gestational age in cases with premature rupture of membrane. pak j Med Sci. 2012;28(1):124-9.

10- El Messidi A, Cameron A. Diagnosis of premature rupture of membranes: Inspiration from the past and insights for the future. J Obstet Gynaecol Can. 2010;32(6):561-9.

11- Di Renzo GC, Roura LC, Facchinetti F, Antsaklis A, Breborowicz G, Gratacos E, et al. Guidelines for the management of spontaneous preterm labor: Identification of spontaneous preterm labor, diagnosis of preterm premature rupture of membranes, and preventive tools for preterm birth. J Matern Fetal Neonatal Med. 2011;24(5):659-67.

12- Cobo T, Palacio M, Martínez Terrón M, Navarro Sastre A, Bosch J, Filella X, et al. Clinical and inflammatory markers in amniotic fluid as predictors of adverse outcomes in preterm premature rupture of membranes. Am J Obstet Gynecol. 2011;205(2):126.e1-8.

13- Lee T, Carpenter MW, Heber WW, Silver HM. Preterm premature rupture of membranes: Risks of recurrent complications in the next pregnancy among a populationbased sample of gravid women. Am J Obstet Gynecol. 2003;188(1):209-13.

14- Bayat MM, Tabrizian F, Dolatabady E, Khalesian B. Analysis of some maternal risk factors in preterm delivery. J Mediv Sci. 2009;3(19):141-6. [Persian]

15- Schleubner E. The prevention, diagnosis and treatment of premature labor. Deut Arztebl Int. 2013;110(13):227-35.

16- Aarts JM, Brons JT, Bruinse HW. Emergency cerclage: A review. Obstet Gynecol Surv. 1995;50(6):459-69.

17- Berghella V, Rafael TJ, Szychowski JM, Rust OA, Owen J. Cerclage for short cervix on ultrasonography in women with singleton gestations and previous preterm birth: A meta-analysis. Obstet Gynecol. 2011;117(3):663-71.

18- Minkoff H, Grunebaum AN, Schwarz RH, Feldman J, Cummings M, Crombleholme W, et al. Risk factors for prematurity and premature rupture of membranes: A prospective study of the vaginal flora in pregnancy. Am J Obstet Gynecol. 1984;150(8):965-72.

19- Mercer BM, Arheart KL. Antimicrobial therapy in expectant management of preterm premature rupture of the membranes. Lancet. 1995;346(8985):1271-9.

20- Kenyon S, Boulvain M, Neilson JP. Antibiotics for preterm rupture of membranes. Cochrane Database Syst Rev. 203;2:CD001058.

21- Steinfeld JD, Lenkoski C, Lerer T, Wax JR, Ingardia CJ. Neonatal morbidity at 34-37 weeks: The role of ruptured membranes. Obstet Gynecol. 1999;94(1):120-3.

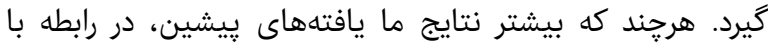

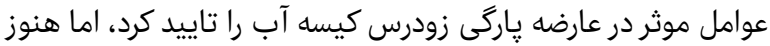

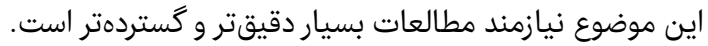

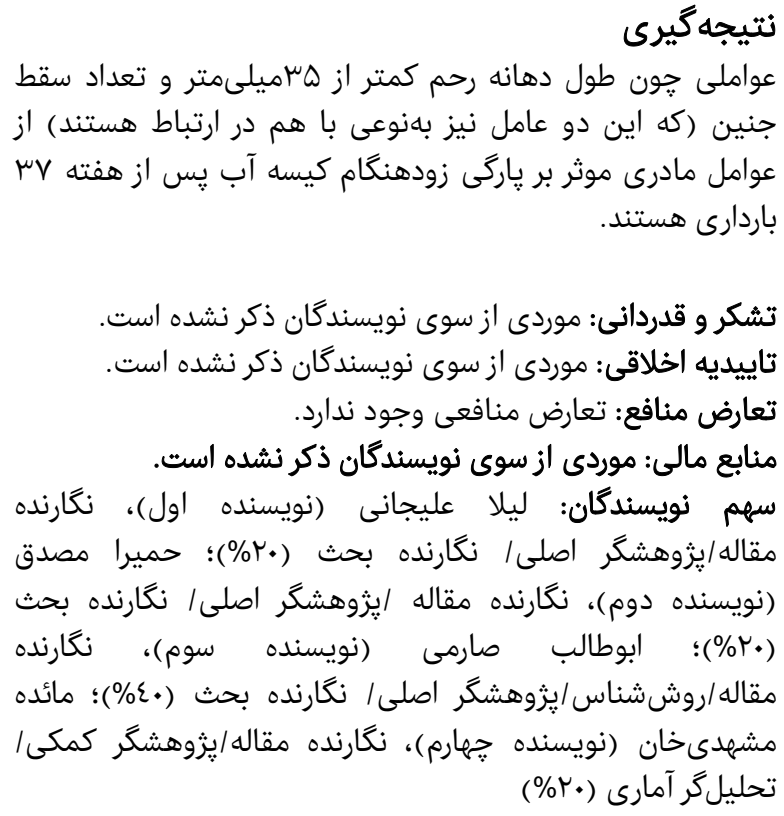

منابع with prelabour rupture of membranes (PROM). University of Witwatersrand Human Research Ethics Committee; 2013.

2- Nili F, Shams Ansari A. Neonatal complications of premature rupture of membrane. Acta Med Iran. 2003;41(3):175-9. [Persian]

3- Li N, Fu Q, Cai W. Cause analysis and clinical management experience of the premature rupture of membrane. 2013;3:222-6.

4- Ahirwar G, Rajput N, Verma YS. Study of patient with premature rupture of membranes. J Evol Med Dent Sci. 2015;4(31):S288-93.

5- Resnik R, Creasy RK, Iams J, Lockwood Ch, Moore Th.

Creasy and resnik's maternal-fetal medicine: Principles and practice. $7^{\text {th }}$ edition. New South Wales: Saunders; 2004.

6- Gahwagi MM, Busarira MO, Atia M. Premature rupture of membranes characteristics, determinants, and outcomes of in Benghazi, Libya. Open J Obstet Gynecol. 2015;5(9):494-504.

7- Choudhary M, Rathore SB, Chowdhary J, Garg S. Pre and post conception risk factors in PROM. Int J Res Med Sci. 